\title{
Highly Charged Hierarchically Structured Porous Anion Exchange Membranes with Excellent Performance
}

\author{
Erigene Bakangura, Congliang Cheng, Liang Wu, Yubin He, Xiaolin Ge, Jin Ran, \\ Kamana Emmanuel and Tongwen $\mathrm{Xu} *$
}

CAS Key Laboratory of Soft Matter Chemistry, Collaborative Innovation Center of Chemistry for Energy Materials, School of Chemistry and Material Science, University of Science and Technology of China, Hefei 230026, P.R. China.

\begin{abstract}
Fabrication of Porous Anion Exchange Membranes for ion transport and ionic selectivity has gained tremendous attention as a prospect for broad application in separation and energy conversion. In this work, we developed a novel strategy to fabricate highly charged hierarchical porous anion exchange membranes (AEM) with high performance. The membranes were prepared by coupling quaternized poly (2dimethylaminoethanol-N-2,3-dimethylphenyl oxide) (QDAPPO) and an organosilane ionic liquid (IL). The electrostatic ionic interactions provide the porous system. When applied in acid recovery, the obtained membranes achieved a high separation factor of 86.5 with proton dialysis coefficient of $0.027 \mathrm{~m} \mathrm{~h}^{-1}$ at $25^{\circ} \mathrm{C}$. This unique efficiency is about 4.7 times that of commercial membrane. More interestingly, the strategy presents in this work tackle the great challenge of fabricating highly charged, thinner and dimensional stable AEM.
\end{abstract}

KEYWORDS: Anion Exchange membrane, hierarchical porous, ion selectivity, ionic liquid, separation

* Corresponding author. Tel: +86(551)-63601587; Fax: +86(551)-63602171; Email: twxu@ustc.edu.cn 


\section{Introduction}

The environmental impact of world population growth is diverse because the rapid demand of food, energy and commodities increases the expansion of industries productions which lead to environmental pollution and rapid decline of the natural resources. Major industries such as metal processing, mining, pulp and paper, paintings, agriculture, vehicles, chemical and food, and electrical power plants produce acidic wastewaters contained toxic heavy metals and detrimental chemicals[1-3]. To ensure sustainable human society development, there is an increasing global concern for the wastewaters treatment within industrial production processes.[4-6] Traditional techniques such as neutralization, crystallization, distillation, thermal decomposition and solvent extraction for wastewater treatments[7-10] focus on purification rather than recovery of resources, and therefore loss enormous amount of energy stored in wastewaters while consuming additional external energy for their operating processes. Membrane based technologies like diffusion dialysis, membrane distillation, electrodialysis, microbial desalination cell, reverse electrodialysis that recover resources and/or energy from wastewaters are more promising for wastewater treatments, sustainable and environmental friendly.[11-18] Diffusion dialysis, an attractive and commercial viable technique due to its low cost and requires minimum energy during operations[5, 19-21], was selected to investigate the performance of AEMs in this work. It is noteworthy that the major challenges of membrane based techniques for wastewater treatment and acid recovery has been the fabrication of cost-effective anion exchange membranes (AEMs) with optimum desired characteristics including high permeation, high selectivity and long-term stability.

In membrane based gradient techniques for acidic wastewater treatment, AEM should selectively allow the passage of anions across the membrane while limiting the transport of the cations and thus to successfully recover acid, the proton $\left(\mathrm{H}^{+}\right)$should cross the AEM taking advantage of its small radii and high mobility than other cations (i.e. $\mathrm{Fe}^{2+}, \mathrm{Cr}^{2+}, \mathrm{Mn}^{2+}$ ). AEMs are usually prepared by attaching cationic groups (i.e. quaternary ammonium (QA), Imidazolium (Im), phosphonium and guanidium) on polymer architectures.[22-24] Compared to their counterpart proton exchange membranes (PEM), the fabrication of high performance AEMs is more challenging because the fundamental characteristics of cationic head groups and the intrinsic 
nature of anions such as low dissociation, low mobility and various shape compared to cations.[25] Firstly, tailoring microphase separated morphology is much easier in PEM than in AEM because the acidity of anionic functional groups is much higher than the basicity of cationic groups. Secondly, low mobility of anions leads to lower ionic transport and poor selectivity of AEMs.[22] A facile straight forward strategy to increase the ionic transport is via increasing the ion exchange capacity (IEC) which unfortunately is usually associated with excessive membrane swelling and compromise of mechanical properties[26]. Thirdly, flexibility of AEMs becomes low as increasing the IEC in rigid aromatic polymers backbone, especially at low humidity that can make the operation failure or maintenance difficulty.[27-30] In order to increase the ion transport and selectivity while preserving the membrane dimensional integrity, previous works attempted to fabricate AEMs with controlled microphase separated morphologies $[31,32]$ or crosslinking polymer chains to enhance the dimensional stability toward high IEC[28, 33-37]. Although these techniques succeed to increase the ionic transport, particularly when external electrical potential is applied for ion transport like in case of electrodialysis, the membrane mass transport is very lower for feasible application in membrane techniques based on concentration gradient like diffusion dialysis. For example, the commercial available DF-120 (a dense AEM with fiber as substrate) has a proton dialysis coefficient $\left(\mathrm{U}_{\mathrm{H}}^{+}\right)$of only $0.009 \mathrm{~m} \mathrm{~h}^{-1}$ and selectivity of 18.5 at $25{ }^{\circ} \mathrm{C}$.

To assure the high ion and mass transport of AEMs, porous membranes have been proposed for versatile applications owing to their high surface area responsible to boost the membrane permeability.[11, 19, 38] Porous asymmetric (Ultra-filtration, UF) membranes prepared via phase inversion routes have been mostly utilized for diffusion dialysis[39, 40]. Porous asymmetric AEMs prepared through immersing brominated poly(phenylene oxide) in multisilicon copolymer solution and in tertiary amine solutions for cationic functionalization followed by sol-gel reaction exhibited an improved $\mathrm{U}_{\mathrm{H}}^{+}$value of $0.020-0.025 \mathrm{~m} \mathrm{~h}^{-1}$ and a separation range from $25-45$ at 25 ${ }^{\circ} \mathrm{C} .[40]$ Very recently, Wang et al[41, 42] reported macroporous asymmetric membrane with high $\mathrm{UH}_{\mathrm{H}}^{+}$values prepared via phase inversion of BPPO crosslinked by $\mathrm{N}, \mathrm{N}$,-tetramethylethylenediamine (TEMED) or poly(ethyleneimine) (PEI). The TPPO-4h membrane (BPPO crosslinked by TEMED) of $1.43 \mathrm{meq} \mathrm{g}^{-1}$ exhibits a high proton dialysis coefficient of $0.043 \mathrm{~m} \mathrm{~h}^{-1}$ and a high separation of 73.8 at $25{ }^{\circ} \mathrm{C}$. 
Consider the obtained high $\mathrm{U}_{\mathrm{H}}^{+}$, the performance of these membranes were counterbalanced with the diffusion of ferrous ions $\left(\mathrm{Fe}^{2+}\right)$ which increased as a results of wide pores and high water uptake. Additionally, porous asymmetric (UF) membranes usually have a thick layer (ca. $>300 \mu \mathrm{m}$ ) responsible for weakening mass transport and sometime lead to operation difficulty. Despite that, fabrication of thin layer porous membranes is still a major challenge limiting the optimization of membrane performance.

In this work, we report a novel strategy to tailor hierarchical mesoporous AEMs (HMPA) with high performance for rapid acid recovery. HMPA takes advantage of the highly charged nanosized pores in gradient structure with a moderate thickness to simultaneously enhance the selectivity and mass transport. To prepare HMPA, poly(2,3-dimethylphenyl-N-(2-hydroxy-N,N-dimethylethanaminium chloride) or quaternized poly(2-dimethylaminoethanol-N-2,3-dimethylphenyl oxide) (QDAPPO) and 3-methyl-1-(3-(triethoxysilyl)propyl)-1H-imidazolium chloride (Ionic liquid, IL) were covalently crosslinked via sol-gel reaction followed by thermal treatment at high temperature. By contrast to reported works, hierarchical porous membranes for gas separation and responsive membranes have been recently prepared by using template method or immersing IL based membranes into ammonium solutions[43-46]. More interestingly, the strategy presents in this work tackle the great challenge of fabricating highly charged AEM with thin layer and good dimensional stability.

\section{Expiremental}

\subsection{Materials}

1-(2-(dimethylamino) ethanol (DMAE) was purchased from J\&K chemicals Ltd and was used without further purification. Sodium sulfates, sodium chloride, Toluene, ethylacetate, NMP, diethyl ether, were purchased from Shanghai Sino Pham Chemical Reagent Co. Ltd. (P. R. China). Bromomethylated (2, 6-dimethyl-1, 4-phenylene oxide) (BPPO) of 52 bromobenzyl (-CH2-Br) was provided by Tianwei Membrane Corporation Ltd.(Shandong, P.R. China). It was purified before use by dissolving in $\mathrm{N}$-methyl-2-pyrrolidone (NMP), precipitating in deionized water, and drying in $30^{\circ} \mathrm{C}$ vacuum oven. Diionized water was used throughout the experiments. 


\subsection{Synthesis of quaternized poly(2-dimethylaminoethanol-N-2,3- dimethylphenyl oxide) (QDAPPO)}

$1.104 \mathrm{~g}(4.2 \mathrm{mmol})$ of 1-(2-(dimethylamino) ethanol (DMAE) was all at once added to a solution of $2 \mathrm{~g}(5.56 \mathrm{mmol}) \mathrm{BPPO}$ in $30 \mathrm{~mL}$ of NMP into $50 \mathrm{~mL}$ bottom flask. The mixture was stirred at room temperature for $16 \mathrm{~h}$ and then poured in toluene to precipitate the product. The precipitate was filtered, washed several times by ethylacetate and final by diethylether and then dried at $40{ }^{\circ} \mathrm{C}$ for $48 \mathrm{~h}$ in air circulating oven. The completed substituted polymer was confirmed by ${ }^{1} \mathrm{H}$ NMR as determined from integration ratio of methylene and aromatic protons.

\subsection{Synthesis of 3-methyl-1-(3-(triethoxysilyl)propyl)-1H-imidazolium chloride (Ion liquid, IL)}

IL was prepared by following the method reported elsewhere[47] and described as follows. 1-Methylimidazole (2.060 g, $25.085 \mathrm{mmol})$ and (3-chloropropyl) triethoxysilane $(6.042 \mathrm{~g}, 25.091 \mathrm{mmol})$ dissolved in toluene $(20 \mathrm{~mL})$ were refluxed for three days. Immediately after stop stirring and heating, viscous oil decant at the bottom of flask and then the toluene can easily removed by pouring. The obtained viscous oil was washed several times by toluene and diethylether anydrous to remove unreacted reagents. The solvents were completely removed under reduced pressure and drying for $24 \mathrm{~h}$ at $60{ }^{\circ} \mathrm{C}$ to afford the title product as viscous oil. The IL is soluble in other polar solvent (i.e.DMSO, Acetonitrile). The chemical structure was confirmed by ${ }^{1} \mathrm{H}$ and ${ }^{13} \mathrm{C}$ NMR (see Figure S1 and S2, ESI)

\subsection{Membrane preparation}

$1 \mathrm{~g}$ of polymer (QDAPPO) was dissolved in $10 \mathrm{~mL}$ of DMSO. Appropriate amount of IL $(10 \%, 20 \%, 30 \%$ and $40 \%$ wt of QDAPPO) was added to the polymer solution at room temperature and allow to mixture for 30 minutes prior to subsequently add TEOS (1:1 ratio corresponding to loaded IL) and 2-3 drop of $1 \mathrm{M} \mathrm{HCl}$ in DMSO. The above mixture was stirred at $45{ }^{\circ} \mathrm{C}$ for $24 \mathrm{~h}$, casted on glass plate and dried at $60{ }^{\circ} \mathrm{C}$ for $12 \mathrm{~h}$ to remove the solvent. The membrane was peered off the glass plate and thermally treated from $70,90,110$ and $120{ }^{\circ} \mathrm{C}$ (1h at each temperature) and at $130{ }^{\circ} \mathrm{C}$ for $3 \mathrm{~h}$ to assure the complete sol-gel reaction and remove of tapped solvent. The obtained thermal treated membrane was immersed in water for $24 \mathrm{~h}$ and then in $2 \mathrm{M}$ 
$\mathrm{NaCl}$ to convert the $\mathrm{Br}$ - ions into $\mathrm{Cl}$ - ions. The membranes were noted as QDA-ILX where $\mathrm{x}$ presents the percentage ratio of IL and TEOS to QDAPPO.
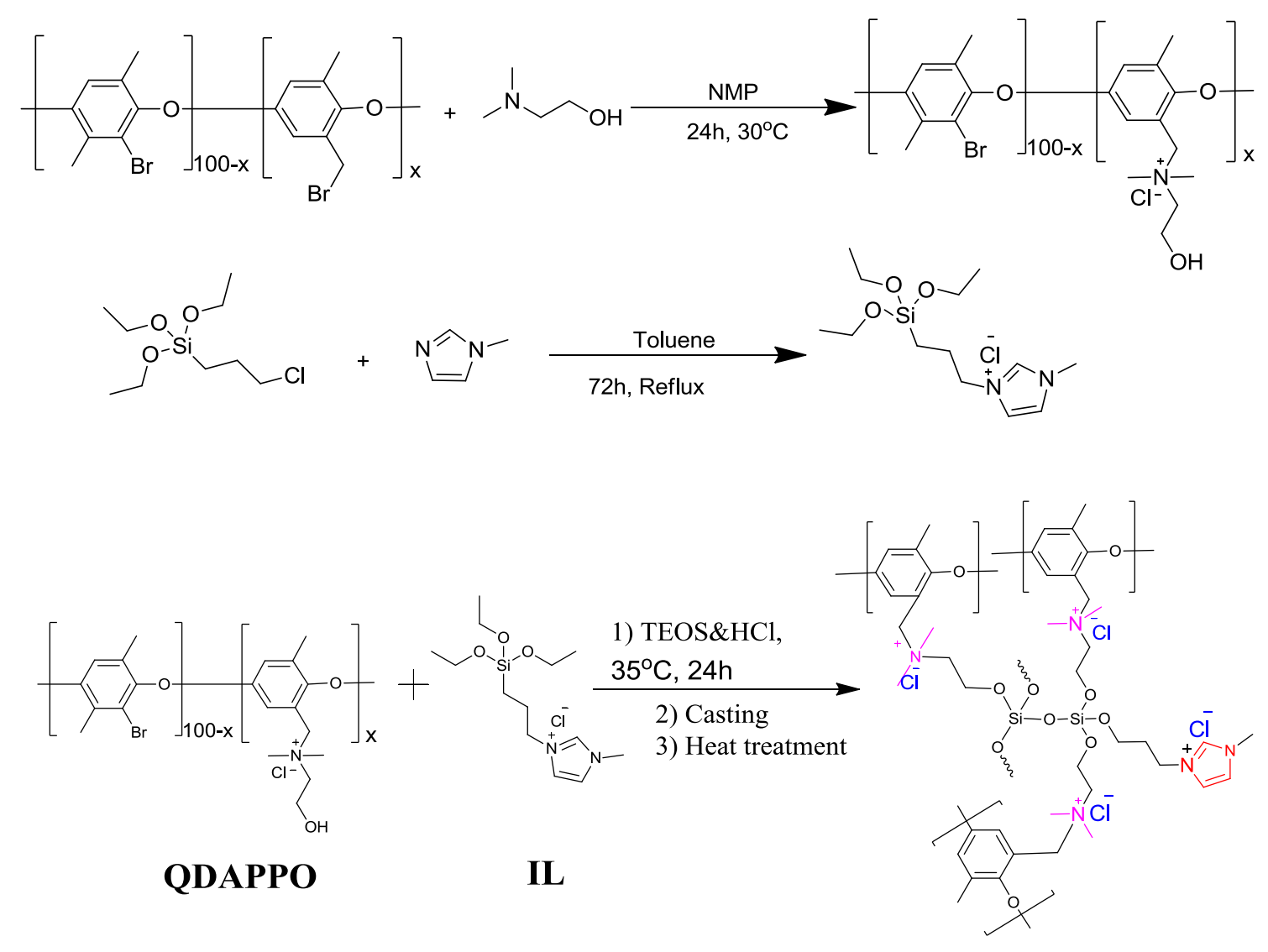

QDA-IL

Scheme 1: Preparation of routes for QDA-IL membranes

\subsection{Polymer and Membrane characterization}

Nuclear Magnetic resonance (NMR) spectra were recorded on a Bruker AVANCEII spectrometer with TMS as an internal standard. Scanning electron microscopy (SEM) images were observed by using scanning electron microscopy (XT30ESEM-TMPHILIP). The samples for SEM (for cross-sectional) were prepared by freezing and breaking samples in nitrogen liquid whereas the sample for surface imaging were prepared by directly cutting a clean membrane into small pieces. Thermal behavior was carried out on NETZSCH STA 449F3 thermogravimetric analyzer (TGA). Samples, pre-dried in vacuum oven at $60{ }^{\circ} \mathrm{C}$ for $24 \mathrm{~h}$, were heated from 50 to $800{ }^{\circ} \mathrm{C}$ at a heating rate of $10^{\circ} \mathrm{C} /$ min under a nitrogen flow. Tensile measurements of membranes were carried out using a dynamic mechanical analyzer (DMA Q800) in controlled force mode using a stretch rate of $0.50 \mathrm{~N} / \mathrm{min}$ at room temperature. Stress-strain curves were 
recorded. Wet state mean that the samples were kept in water prior to testing whereas dry state mean that the samples were dried in vacuum oven before the testing to remove any trace of water.

Ion exchange capacity, Water uptake and swelling ratio were characterized in the same way as our published papers[48].

Diffusion dialysis tests were performed in a cell of two chambers where are separated by an AEM with an effective area of $5.73 \mathrm{~cm}^{2}$. Assimilated acid wastewater solution filled in feed chamber at $100 \mathrm{~mL}$ volume contains $1 \mathrm{~mol} \mathrm{~L}$ 1 of $\mathrm{HCl}$ and $0.2 \mathrm{~mol} \mathrm{~L}^{-1}$ of $\mathrm{FeCl}_{2}$ mixed solution. The permeate chamber was filled with distilled water at $100 \mathrm{~mL}$. Prior to the test, the AEMs were conditioned in feed solution for $24 \mathrm{~h}$ and washed thoroughly with water before each test. Mechanical electrical stirrers were used to stir both chambers synchronously and identically to minimize concentration polarization effects. After $45 \mathrm{~min}$ of diffusion, $\mathrm{HCl}$ concentration diffused in permeate solution were determined by using standard titration method with $\mathrm{Na}_{2} \mathrm{CO}_{3}$ solution using methyl orange as the indicator. The concentration of $\mathrm{FeCl}_{2}$ was measured by using Inductively Coupled Plasma Mass Spectrometry (ICP-MS). Dialysis coefficient can be calculated from the concentration of species by the following equation:

$$
U=\frac{M}{A t \Delta C}
$$

Where $\mathrm{M}$ is the amount of the component transported in moles, $\mathrm{A}$ is the effective area in square meters; $\mathrm{t}$ is the time in $\mathrm{h}$, and $\Delta \mathrm{C}$ is the logarithmic average concentration between the two chambers in mole per cubic meter as in equation below:

$$
\Delta C=\frac{\left(c_{f}^{0}-c_{d}^{t}-c_{f}^{t}\right)}{\operatorname{Ln}\left[\left(c_{f}^{0}-c_{d}^{t}\right) / c_{f}^{t}\right]}
$$

Where $C_{f}^{\circ}$ and $C_{f}^{t}$ are the feed concentrations at time 0 and t, respectively, and $C_{d}^{t}$ the dialysate concentration at time t. It should be noted that $\left(C_{f}^{o}-C_{d}^{t}-C_{f}^{t}\right)$ is not equal 
to zero, because water transporting through the membrane will cause volume changes in the cell chambers during the experiment.

The separation factor ( $\mathrm{S}$ ) between $\mathrm{HCl}$ and $\mathrm{FeCl}_{2}$ is defined as a ratio of the dialysis coefficients of protons $\left(\mathrm{UH}^{+}\right)$and of cationic iron $\left(\mathrm{UFe}^{2+}\right)$.

$$
S=\frac{U_{H^{+}}}{U_{F \varepsilon^{2+}}}
$$

\section{Results and Discussions}

\subsection{Synthesis and characterization of QDA-IL AEMs}

Scheme 1 displays the preparation route of DA-IL AEMs. First, the QDAPPO was synthesized through reacting BPPO with 1-(2-(dimethylamino)ethanol (DMAE). The initial molecular weight of BPPO was maintained throughout the synthesis since the reaction was carried at room temperature and in mild medium. After purification, the polymer structure was confirmed by the ${ }^{1} \mathrm{H}$ NMR and FTIR spectroscopy as shown in Figure 1 and Figure S3 (ESI). The proton characteristic peak at $4.34 \mathrm{ppm}$ assigned to brominated methylene of BPPO was used to monitor the substitution reaction and determine the grafting degree. As clearly shown in Figure 1, the substituted methylene proton peaks shift to $4.5 \mathrm{ppm}$ which indicates the successful reaction of BPPO with DMAE. The absence of any proton signals around $4.34 \mathrm{ppm}$ suggests the fully substituted brominated methylene. The substitution degree was calculated as the ratio of the integration of the peak at $4.5 \mathrm{ppm}$ and the peaks at 7-6.5 ppm. Further, the QDAPPO is confirmed by the proton characteristics peaks of $\mathrm{N}-\mathrm{CH}_{2}-\mathrm{CH}_{2}-\mathrm{OH}$; the methylene protons of $-\mathrm{N}-\mathrm{CH}_{2}-$ and $-\mathrm{CH}_{2}-\mathrm{OH}$ signal at $3.5 \mathrm{ppm}$ and $3.9 \mathrm{ppm}$, respectively. Hydroxyl groups (-C-OH) are absolutely confirmed by the proton signal at $5.94 \mathrm{ppm}$. It is worth to note here that the QDAPPO has versatile solubility in several conventional solvents for membrane preparation (i.e. DMSO, Methanol, and DMF). The FTIR spectrum of QDAPPO is shown in Figure S3. The characteristics absorption bands of the QDAPPO apparently appear at 3500-3000 $\mathrm{cm}^{-1}$ (broad band, hydrogen bonding among -OH groups) and 1092-1088 $\mathrm{cm}^{-1}$ (symmetric stretching of C-N). While the disappearance of the BPPO characteristics band in finger-print region at $738-735 \mathrm{~cm}^{-1}$ corresponding to $-\mathrm{CH}_{2} \mathrm{Br}$ confirms a complete substitution reaction. 


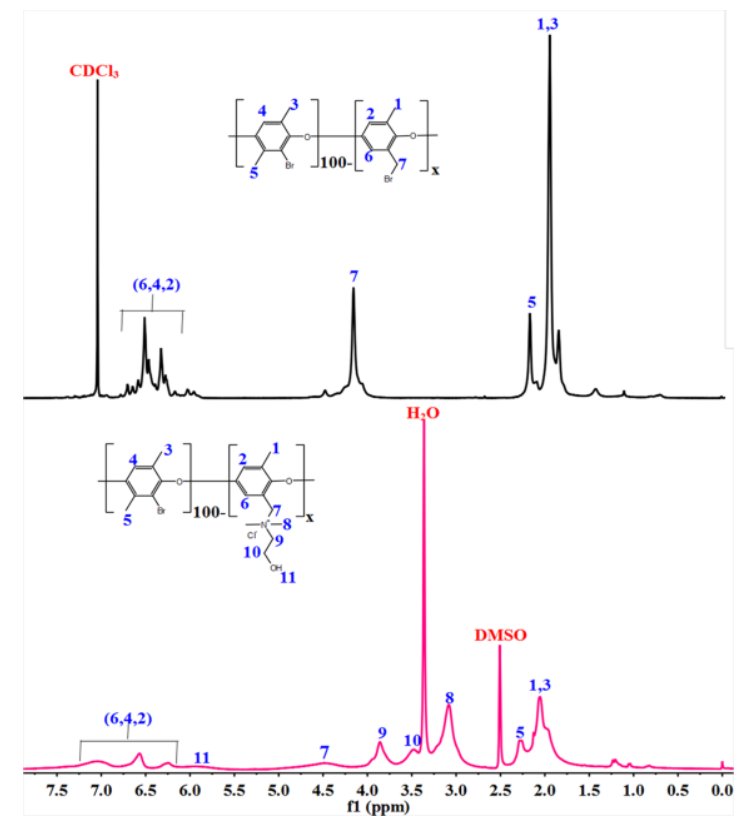

Figure 1: a) ${ }^{1} \mathrm{H}$ NMR spectra of BPPO (in $\mathrm{CDCl}_{3}$ ) and QDAPPO (in DMSO-d6)
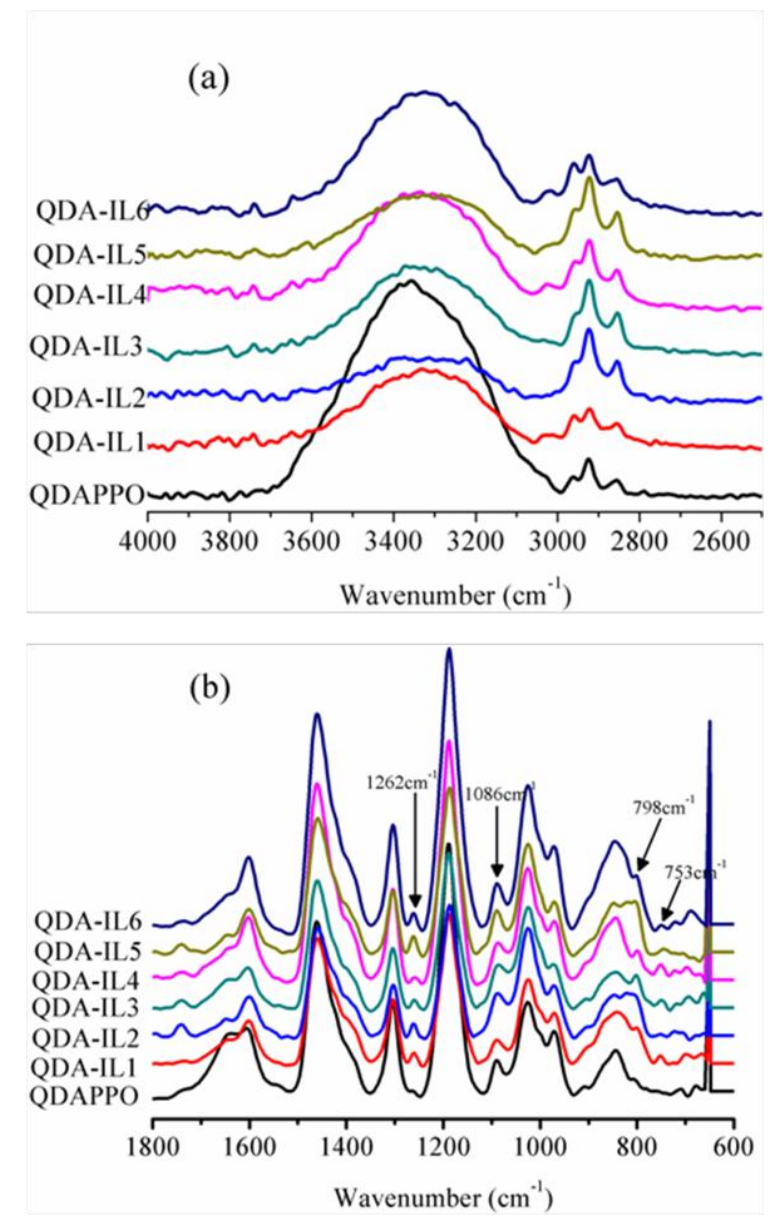

Figure 2: FTIR spectra of QDAPPO and QDA-IL membranes; a) 4000-2500 $\mathrm{cm}^{-1}$ and b) $1800-600 \mathrm{~cm}^{-1}$. 
DA-IL AEMs were prepared by grafting the QDAPPO and IL through sol-gel reaction. The successful completion of sol-gel reaction was monitored by the FTIR spectroscopy (Figure 2a-b). The absorption bands at 2962-2849 $\mathrm{cm}^{-1}$, characteristics of methyl and methylene, become stronger than those of QDAPPO due to incorporation of IL bearing $-\mathrm{CH}_{2}$ - and $-\mathrm{CH}_{3}$. The absorption bands at $1086 \mathrm{~cm}^{-1}$ rise in intensity and become wider because the anti-symmetric stretching of Si-O-C and $\mathrm{Si}-\mathrm{O}-\mathrm{Si}$ bond while the band at $798 \mathrm{~cm}^{-1}$ indicates the vibration of $v \mathrm{Si}-\mathrm{O}-\mathrm{Si}$.[49, 50]. The absorption band at $1260 \mathrm{~cm}^{-1}$ appears in QDA-IL membranes arise from the stretching vibration of $\mathrm{C}-\mathrm{N}$ in imidazolium cation ring and the absorption band at 753 $\mathrm{cm}^{-1}$ confirms the vibration mode of imidazolium cations.[30] The broad band at $3500-3000 \mathrm{~cm}^{-1}$ are assigned to hydrogen bond among unreacted $\mathrm{Si}-\mathrm{OH}$ and trace of water in the QDA-IL membranes are weakened relative to QDAPPO because most of the $\mathrm{C}-\mathrm{OH}$ groups were reacted.

\subsection{Membrane Morphology}

The morphologies of the membranes were observed by scanning electron microscopy (SEM). As illustrated in Figure 3, interconnected three-dimensional porous structures were formed. The cross-sectional SEM images shows hierarchically distributed nanopores along the cross-sectional of the membrane. It is apparent that the QDA-IL membranes possess nanoporous structure for which the pores density and dimensions increase with increasing the IL contents. SEM images of QDA-IL1 to QDA-IL6 membranes captured on both the surface and cross-sectional are illustrated in Figure S4 and S5 (Supporting information). All QDA-IL membranes show porous structure except QDA-IL1 membrane which shows a compact surface. Membranes with different thicknesses $(43-59.9 \mu \mathrm{m})$ were obtained due to variation of concentrations of casted solutions. The segregation of imidazolium cationic groups and their electrostatic ionic complexation with alkoxides formed during silica particles formation provided the hierarchical porous system. Consequently, the pores are cationic charged by imidazolium groups that would influence the selectivity of the membranes. Recently, a number of reported work revealed similar hierarchical pores formation when the IL complexes with anions[43, 44] via immersing partial dried membranes into anion solutions. In contrast to asymmetrical porous membranes prepared through phase inversion method, whose cross-sectional pores consists of macroporous and thick layer, the hierarchical porous structure having well-defined 
interconnected pores across the membrane and low thickness can increase the tortuosity of ion transfer to improve the selectivity of the membranes and as well enhance the mass transport
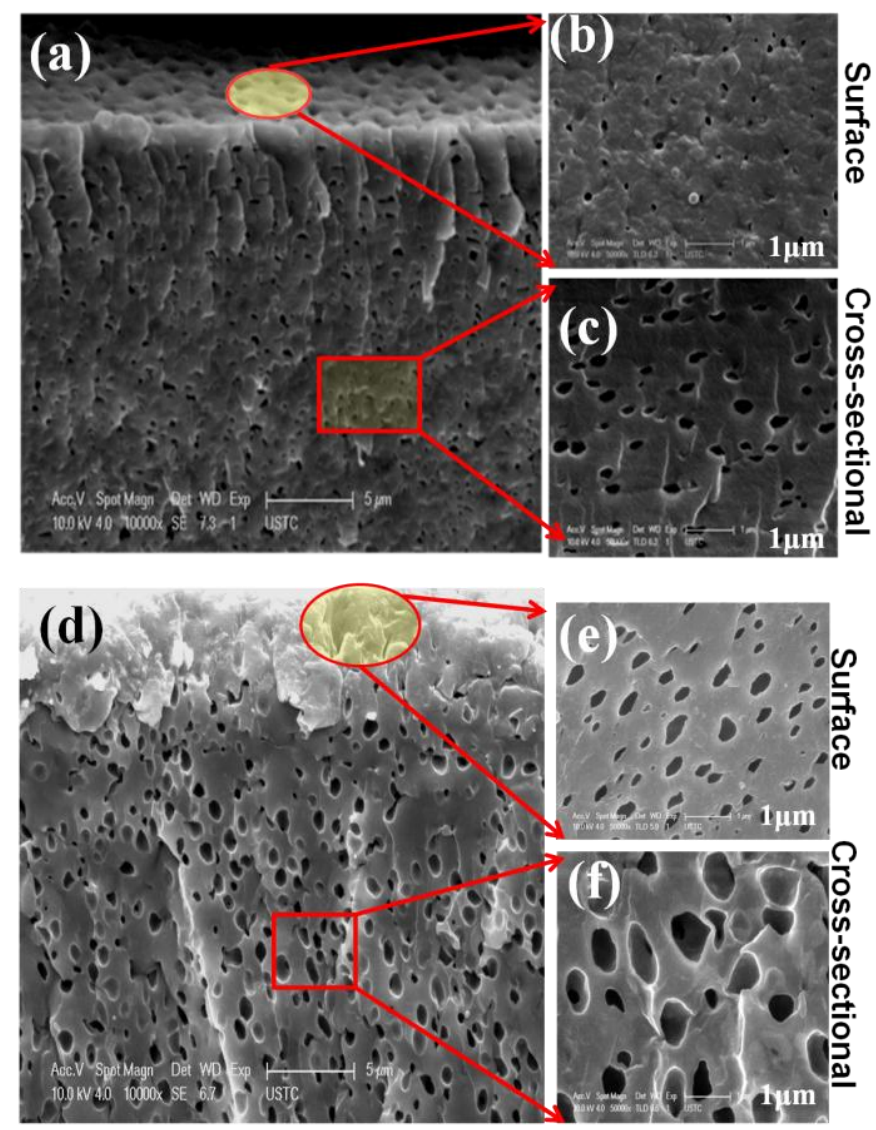

Figure 3: SEM images of QDA-IL members; QDA-IL2 (a-c) and QDA-IL6 (d-e)

\subsection{Ion Exchange Capacity}

IEC is a primary parameter to consider for preparing and characterizing the AEMs since the transport of anions across the membranes relies mostly on the IEC due to the low mobility of anions. In this work, the IECs of QDA-IL membranes were optimized through increasing the density of cationic functional groups; quaternary ammonium from QDAPPO and imidazolium from IL. The theoretical and experimental IECs are shown in Table 1. The difference between the theoretical and experiments is attributed to the low mobility of $\mathrm{Cl}^{-}$ion and its slow exchanging with the sulphate ions during the IEC measurement. Compared to the QDAPPO membrane, the IECs of DA-IL membranes increased significantly with addition of the IL and attain an experimental high value of $2.49 \mathrm{mmol} \mathrm{g}^{-1}$ at $25{ }^{\circ} \mathrm{C}$. The low IEC of QDA-IL1 is 
attributed to reduced membrane flexibility caused by crosslinking between hydroxyl groups of polymer and silane $(\mathrm{C}-\mathrm{OH}$ and $\mathrm{Si}-\mathrm{OH})$.

Table 1: Membrane composition, thickness, ion exchange capacity (IEC) and water uptake (WU)

\begin{tabular}{|c|c|c|c|c|c|c|c|}
\hline \multirow[t]{2}{*}{ Membrane } & \multirow{2}{*}{$\begin{array}{l}\text { IL+TEOS to } \\
-\mathrm{OH}(\%)\end{array}$} & \multicolumn{2}{|c|}{ Thickness $(\mu \mathrm{m})$} & \multicolumn{2}{|c|}{ IEC (meq/g) } & \multicolumn{2}{|c|}{ WU (\%) } \\
\hline & & Dry $^{a}$ & Wet & IEC $^{\mathbf{b}}$ & IEC $^{\mathrm{c}}$ & $25^{\circ} \mathrm{C}$ & $60^{\circ} \mathrm{C}$ \\
\hline QDAPPO & 0 & 55.7 & 66.2 & 2.13 & 2.11 & 63.76 & 69.1 \\
\hline QDA-IL-1 & 10 & 55.1 & 63 & 2.37 & 2.13 & 51.25 & 52.7 \\
\hline QDA-IL-2 & 20 & 43.3 & 76 & 2.47 & 2.20 & 66.2 & 72.8 \\
\hline QDA-IL-3 & 40 & 56.6 & 81 & 2.55 & 2.24 & 83.92 & 88.0 \\
\hline QDA-IL-4 & 60 & 53.6 & 92.8 & 2.70 & 2.38 & 130.8 & 135.2 \\
\hline QDA-IL-5 & 80 & 59.9 & 101 & 2.83 & 2.42 & 141.1 & 147.6 \\
\hline QDA-IL-6 & 100 & 59.6 & 107 & 2.93 & 2.49 & 157.9 & 163.3 \\
\hline
\end{tabular}

${ }^{\mathrm{a}}$ Determined from SEM imaging, ${ }^{\mathrm{b}}$ Theoretical IEC, ${ }^{\mathrm{c}}$ Experimental IEC determine by using titration method,

\subsection{Water Uptake (WU)}

The Water uptake (WU) has a profound effect on the transport behavior of ions across the membrane and as well on dimensional and mechanical stability. Rationally, the high WU of the ion exchange membrane would be associated with high ion transport except that it usually undermines the selectivity of the membranes. The DA-IL membranes have the water uptake ranging from 51.25 to $157.92 \%$ at $25^{\circ} \mathrm{C}$ (Table 1). This range is reasonably viable for the DD and several other AEMs based applications. To emphasize, the WU of QDA-IL membranes are interestingly lower than that of asymmetrical porous BPPO modified TEMED[41] (ca. $330 \%$ ). The cationic groups from IL,silica particles and free volume in pores contributed on increasing the WU of QDA-IL membranes. Thermal annealing having initiated covalent crosslinking (Si-OC) among silica and hydroxyl groups of QDAPPO decreased the membrane flexibility and consequently diminishes the WU of QDA-IL1 despite an observed slight increase on IEC. To test the swelling behavior, the DA-IL membranes were immersed in water and heated at $65{ }^{\circ} \mathrm{C}$ for $24 \mathrm{~h}$. Hence, The WU increased as increasing the contents of IL. As shown in Table.1, the water uptake increases slightly as the temperature increases from $25{ }^{\circ} \mathrm{C}$ to $65{ }^{\circ} \mathrm{C}$ while all membranes maintained the initial dimensional 
stability. Usually, the WU value of AEM increases with temperature as a result of increased polymer chain flexibility which frees the cationic groups to fully adsorb the water molecules.

\subsection{Diffusion dialysis performance}

QDA-IL membranes were used in DD to evaluate their performance for application in inorganic acid recovery. The model acidic wastewater solution used in this work contains the mixture of aqueous $\mathrm{HCl}$ and $\mathrm{FeCl}_{2} \cdot[41,51]$ This wastewater solution was preferably chosen because the $\mathrm{Cl}^{-}$and $\mathrm{Fe}^{2+}$ ions are not only abundant in wastewaters but the $\mathrm{Fe}^{2+}$ ion has also a small ionic radius among the metal pollutants. Therefore, the effective performance of the AEMs in the above model solution would suggest certainly their performance in several other applications such as DD power, MDC and RED,etc.

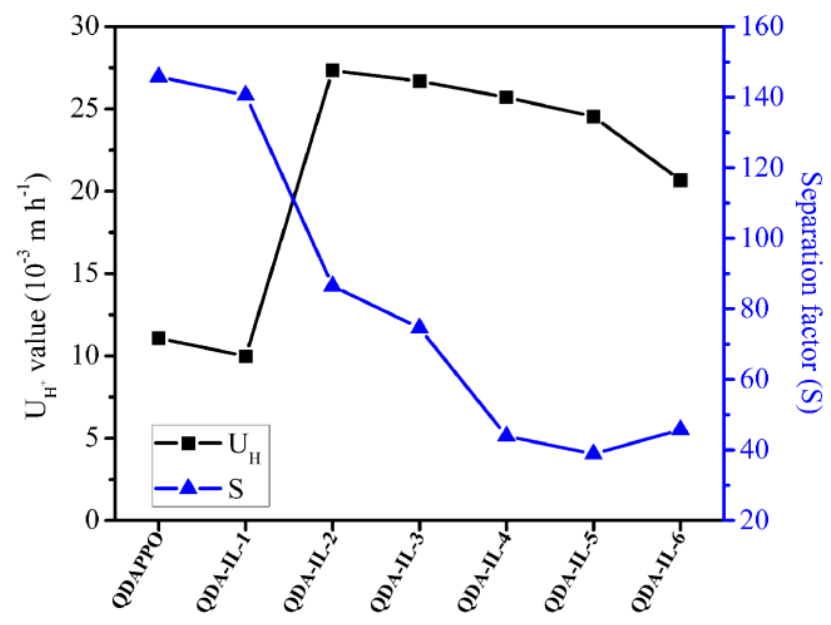

Figure 4: Proton dialysis coefficient $\left(\mathrm{UH}^{+}\right)$and separation factor (S) of QDAPPO and QDA-IL membranes at $25^{\circ} \mathrm{C}$ 


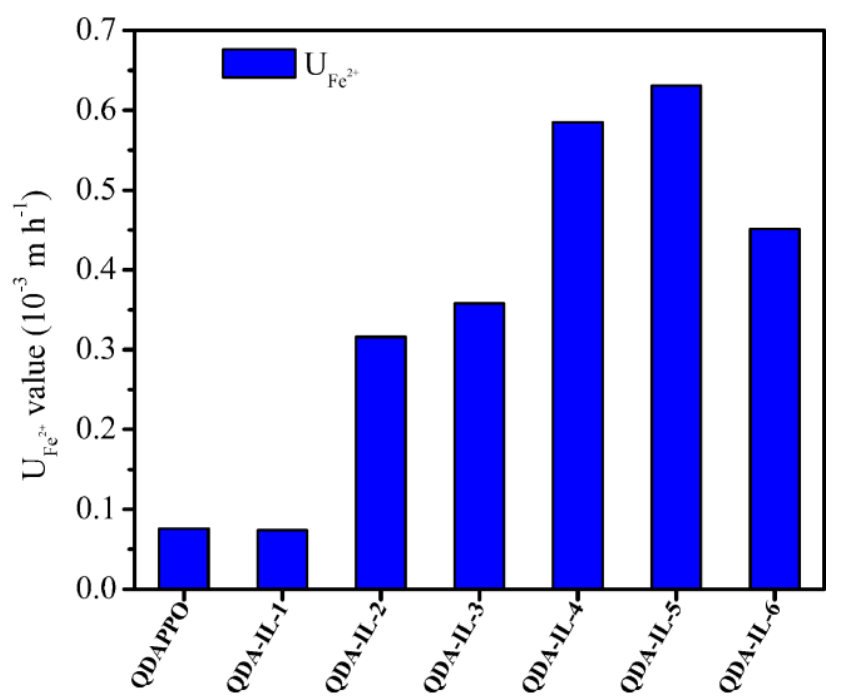

Figure 5: Ferrous ion dialysis coefficient $\left(\mathrm{U}_{\mathrm{Fe}}{ }^{2+}\right)$ of QDAPPO and QDA-IL membranes at $25^{\circ} \mathrm{C}$

Figure 4 shows the proton dialysis coefficient $\left(\mathrm{UH}^{+}\right)$and separation factor $(\mathrm{S})$ of QDA-IL and QDAPPO membranes at $25^{\circ} \mathrm{C}$. The $\mathrm{U}_{\mathrm{H}}{ }^{+}$values of QDA-IL membranes are in the range of $0.020-0.0273 \mathrm{~m} \mathrm{~h}^{-1}$ at $25{ }^{\circ} \mathrm{C}$ except for QDA-IL1 which exhibits low $\mathrm{UH}^{+}$value of $0.0098 \mathrm{~m} \mathrm{~h}^{-1}$ while the $\mathrm{U}_{\mathrm{H}}{ }^{+}$value of QDAPPO is $0.011 \mathrm{~m} \mathrm{~h}^{-1}$ at the same conditions. Compared to some reported AEMs, $\mathrm{UH}^{+}$values of QDA-IL membranes are about 3 times the value of commercial DF-120 $\left(0.009 \mathrm{~m} \mathrm{~h}^{-1}\right.$ at $\left.25{ }^{\circ} \mathrm{C}\right)$ and quaternized PPO-SiO 2 membranes[52] (0.005-0.011 $\mathrm{m} \mathrm{h}^{-1}$ at $\left.25{ }^{\circ} \mathrm{C}\right)$, and 2 times higher than the PVA and multi-alkoxy-silicon copolymer blending membranes[48, 53] (0.01-0.017 $\mathrm{m} \mathrm{h}^{-1}$ at $\left.25^{\circ} \mathrm{C}\right)$. The $\mathrm{UH}_{\mathrm{H}}{ }^{+}$trend in QDA-IL membranes first decreases with addition of $10 \%$ of the IL and then increases upon adding $20 \%$ of IL. Compared to QDAPPO, the high $\mathrm{UH}_{\mathrm{H}}{ }^{+}$values of QDA-IL2 to QDA-IL6 membranes are attributed to different membrane morphological structure (dense $v s$. hierarchical porous), different mechanical properties (rigidity vs. elasticity) and chemical compositions (crosslinked -C-OH groups vs Si-O-Si bonds). Free hydroxyl groups can easily form the hydrogen bond with proton which can accelerate the transport of $\mathrm{HCl}$ across the membrane whereas crosslinked $-\mathrm{OH}$ groups make the membrane rigid and tough. When only $10 \%$ of the IL was added, a certain amount of hydroxyl groups of QDAPPO were consumed into sol-gel reactions and through thermal annealing the unreacted hydroxyl groups undergo thermal crosslinking to form a rigid dense membrane. As a result, unreacted hydroxyl groups in QDA-IL1lack sufficient flexibility to facilitate the diffusion of protons at $25{ }^{\circ} \mathrm{C}$ as evidenced from the DMA result (Figure 8). Upon 
adding $20 \%$ of IL, the hierarchical porous system was formed and the Si-O-Si bond increased into the membrane matrix. This contributed to the high $\mathrm{UH}_{\mathrm{H}}^{+}$value of QDAIL2 membrane. Despite the increase of WU with increasing the IL content, there is a decreasing trend in $\mathrm{UH}^{+}$values observed from QDA-IL2 to QDA-IL6. This trend is explained by: i) the increase of cationic groups reduce the proton diffusion because the increased friction and electrostatic repulsion[54, 55] ii) Addition of IL is marked with increase of silica particles which significantly facilitate the protons adsorption rather than desorption as a results of increased bound water.[56] iii) the competition between $\mathrm{H}^{+}$and $\mathrm{Fe}^{2+}$ ions transport in the membranes as the pore size increases since the $\mathrm{Fe}^{2+}$ ion has large radii and higher valence state than $\mathrm{H}^{+}$ion as previously reported[48]. Consequently, the $\mathrm{U}_{\mathrm{H}}{ }^{+}$value decreases consistently from the QDA-IL2 to QDA-IL6. Meanwhile, as shown in Figure 5, the $\mathrm{UFe}^{2+}$ value increases progressively with increasing the IL content as a result of increased WU and pores density. This result is in accord with general trend of $\mathrm{UFe}^{2+}$ variation with WU. Further comparison with some reported AEMs, shows that the $\mathrm{UFe}^{2+}$ of QDA-IL2 is about 2.2, 1.8-6 and 1.5 times the $\mathrm{UFe}^{2+}$ value of porous asymmetrical BPPO modified by multisilicon membrane[40] $\left(0.000699 \mathrm{~m} \mathrm{~h}^{-1}\right)$, porous asymmetrical membrane BPPO modified by various amines[41, 42] $\left(0.000582-0.002 \mathrm{~m} \mathrm{~h}^{-1}\right)$ and commercial DF-120 $\left(0.000486 \mathrm{~m} \mathrm{~h}^{-1}\right)$, respectively. The decrease of $\mathrm{U}_{\mathrm{Fe}}{ }^{2+}$ value from QDA-IL5 to QDA-IL6 is attributed to increase of charge density inside the pores. At first, the $\mathrm{U}_{\mathrm{Fe}}{ }^{2+}$ value increases progressively as a result of increasing pores density and dimension in QDA-IL membranes. The addition of the IL in QDA-IL membranes would possibly reach a threshold of pores density and dimensions and therefore further addition of IL would alternatively increase more the pores charge density. Since the $\mathrm{Fe}^{2+}$ ion transport is defined mainly by the adsorption rather than diffusion, the charge density inside the pores would greatly impact the cations transport despite the increase of WU. It is essential to emphasize here that WU in QDA-IL membranes is defined by the cationic groups and bound water associated with adsorption of hygroscopic silica particles. The bound water in silica particles would have a trivial effect on the $\mathrm{U}_{\mathrm{Fe}}{ }^{2+}$ value because the $\mathrm{Fe}^{2+}$ ion is large in size to traverse the interstitial of silica particles. The QDAPPO and QDA-IL1 exhibit the lowest $\mathrm{UFe}^{2+}$ values of $0.000076 \mathrm{~m} \mathrm{~h}^{-1}$ and $0.000074 \mathrm{~m} \mathrm{~h}^{-1}$ which are attributed to compactness of the membranes initiated from crosslinking among hydroxyl groups after heat treatment. This indicates that the water 
molecules are mainly bound on cationic groups which selectively allow the passage of anions (i.e. $\mathrm{Cl}^{-}$). As a result of diffusion coefficients, the QDAPPO records the highest separation factor (S) of 145.78 which decreases upon addition of IL. From QDA-IL2 to QDA-IL6, the S decreases from 86.51 to 38.8, respectively. Despite its highest S, the mass transfer of QDAPPO is very low as indicated with the $\mathrm{UH}_{\mathrm{H}}^{+}$values. By contrast, the QDA-IL2 to QDA-IL6 exhibit viable and high mass transfer for acid recovery. Compared to some reported $\mathrm{AEMs}$ for acid recovery using $\mathrm{HCl}$ and $\mathrm{FeCl}_{2}$ as model acidic wastewater solution, the hierarchical porous QDA-IL2 and QDA-IL3 membranes have excellent selectivity and superior S. For example, as shown in Fig.6, the QDA-IL2 exhibits an $\mathrm{S}$ of 86.5 at $25{ }^{\circ} \mathrm{C}$ which is about 9.6 times the $\mathrm{S}$ of commercial DF-120, suggesting the ultra-selectivity of QDA-IL membranes for acid recovery.

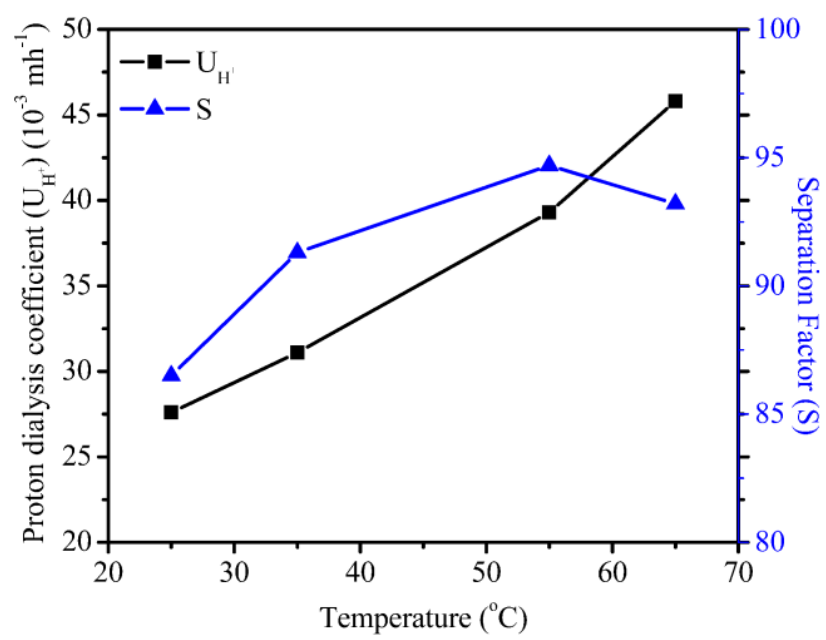

Figure 6: Proton dialysis coefficient $\left(\mathrm{U}_{\mathrm{H}}{ }^{+}\right)$of QDA-IL2 membrane at different temperature

QDA-IL2 was used to investigate the performance of diffusion dialysis at different temperatures. The results of proton diffusion coefficients and separation factor (S) are shown in Figure 6. The $\mathrm{UH}^{+}$of QDA-IL2 increases from 0.027 to $0.045 \mathrm{~m} \mathrm{~h}^{-1}$ with increasing temperature from 25 to $55{ }^{\circ} \mathrm{C}$. This is attributed to the increase of protons mobility with temperature. Concurrently, its separation factor slightly increases from 86.5 to 94.7 when increases temperature from $25{ }^{\circ} \mathrm{C}$ to $55{ }^{\circ} \mathrm{C}$, respectively. Hence, further increase of temperature to $65^{\circ} \mathrm{C}$ drops slightly the separation factor to 93.2. Despite the gradually increase of $\mathrm{UH}_{\mathrm{H}}{ }^{+}$with temperature, the high temperature increases the water mobility which facilitates as well more significantly the diffusion 
of $\mathrm{Fe}^{2+}$ ions across the membrane. Given that the diffusion dialysis mostly performs at ambient temperature, the QDA-IL2 is an excellent choice for acid recovery.

\subsection{Thermal stability}

The thermal stability of the QDAPPO and QDA-IL membranes were evaluated by TGA under a nitrogen atmosphere. Obviously, the incorporation of IL enhances the thermal stability of QDA-IL membranes. From Figure 7a, the first decomposition started from low temperature (ca. $50-120{ }^{\circ} \mathrm{C}$ ) is attributed to the removal of bounding water molecules in the membrane. This decomposition is much more significant for the QDAPPO membrane. The second weight loss onset at $178{ }^{\circ} \mathrm{C}$ for the QDA-IL membranes and at $141{ }^{\circ} \mathrm{C}$ for QDAPPO membrane is attributed thermal decomposition of quaternized amines. As clearly shown in Figure 7b, the derivative degradation weights of QDAPPO shows a peak at $176{ }^{\circ} \mathrm{C}$ indicating the maximum decomposition of quaternary amines whereas the QDA-IL membranes, combining both quaternary amines and imidazolium cations, exhibit maximum cationic group decompositions at $234{ }^{\circ} \mathrm{C}$. Further comparing the QDA-IL membranes, the peak at $234{ }^{\circ} \mathrm{C}$ increases in intensity as increasing the IL content and rather shifts to high temperature for QDA-IL5 and QDA-IL6 membranes due to higher thermal stability of the imidazolium cations than the quaternary amines.[57] The last weight loss onset at $379{ }^{\circ} \mathrm{C}$ is attributed to the thermal decomposition of covalent bonds $(\mathrm{C}-\mathrm{OH}, \mathrm{Si}-\mathrm{O}-\mathrm{Si}$ and $\mathrm{Si}-\mathrm{O}-\mathrm{C}$ ) and the cleavage of polymer backbones.
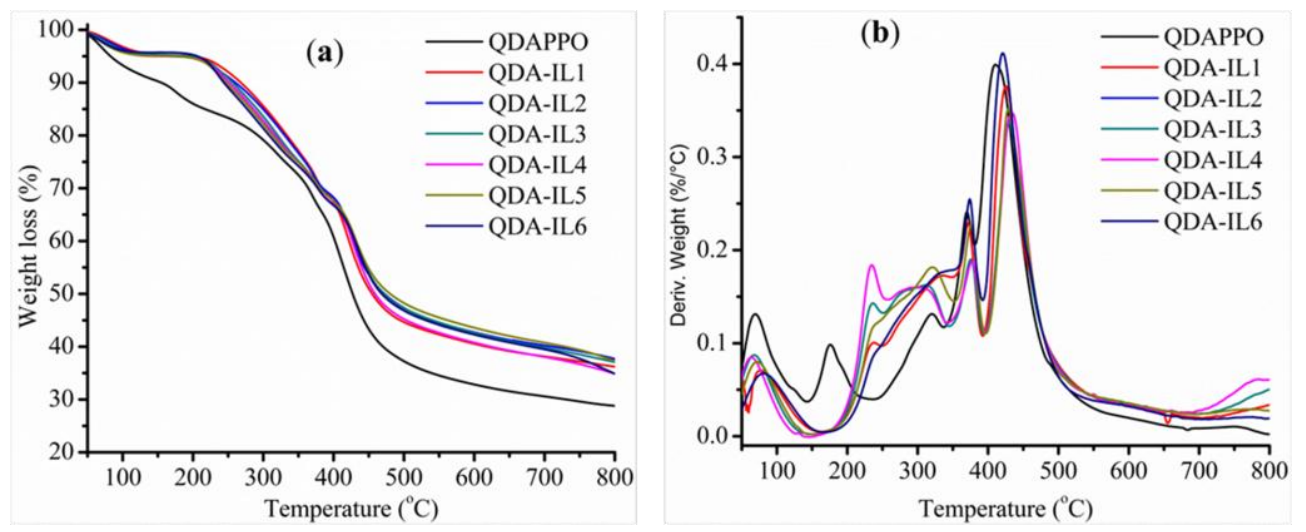

Figure 7: Thermogravimetric graphs of QDAPPO and QDA-IL membranes; a) percentage weight loss vs. temperature b) derivative weight (mg) vs. temperature

\subsection{Mechanical properties}


Mechanical stability is usually one of the critical challenges when the AEMs with high IEC are desired. In most cases, the high IEC leads to excessive membrane swelling and compromises the mechanical stability of AEMs due to weak interaction among the polymer chains. The mechanical properties of DA-IL membranes in both wet (membrane kept in water prior to testing) and dry states are shown in Figure 8a and b. It is apparent in general trend that the incorporation of IL into QDAPPO enhanced the mechanical properties. In dry states, upon incorporating IL into QDAPPO, the tensile strength (TS) and elongation at break (EB) firstly decrease and then increases (Figure 8a). As shown in Figure 8a, the dry QDA-IL membranes displayed TS and EB of 11-28.6 MPa and 3.3-12.6 \% while the QDAPPO has the TS and EB of 14.9 MPa and $4.92 \%$, respectively. The addition of IL within QDAPPO matrix firstly weakens the strength and elongation at $10 \%$ wt of IL and then further addition of IL enhances remarkably the EB, especially in wet conditions. The best explanation is that the introduction of IL at first decreases the hydroxyl groups which can maintain the membrane flexibility in dry condition owing to hydrogen bonds. In wet conditions, the EB increases with addition of IL up to QDA-IL2 membrane and then decreases from QDA-IL 3 to QDA-IL6 as a result of increasing pores density by IL in QDA-IL membranes. As the IL contents increases, the QDA-IL1 and QDA-IL2 gained the flexibility as a result of moderate crosslinking. Further addition of IL increases the pores density (free volume) in membrane matrix and consequently decreases the flexibility. In other words, the high density of pores in membrane matrix weakens the elongation at break. Compared the EB of membranes in both dry and wet condition (Figure 8b), there is a remarkable increase between the couple of QDA-IL membranes than in QDAPPO. This implies that WU has great impact on QDA-IL membranes. For example, the elongation at break of QDA-IL1 increased about 9 folds from $3.2 \%$ to $28.3 \%$ while QDA-IL2 increases from $6.11 \%$ to $46.47 \%$, respectively.. In comparison to QDAPPO, the QDA-IL membranes are more flexible and tough in wet conditions which make them more suitable for various AEM applications. 

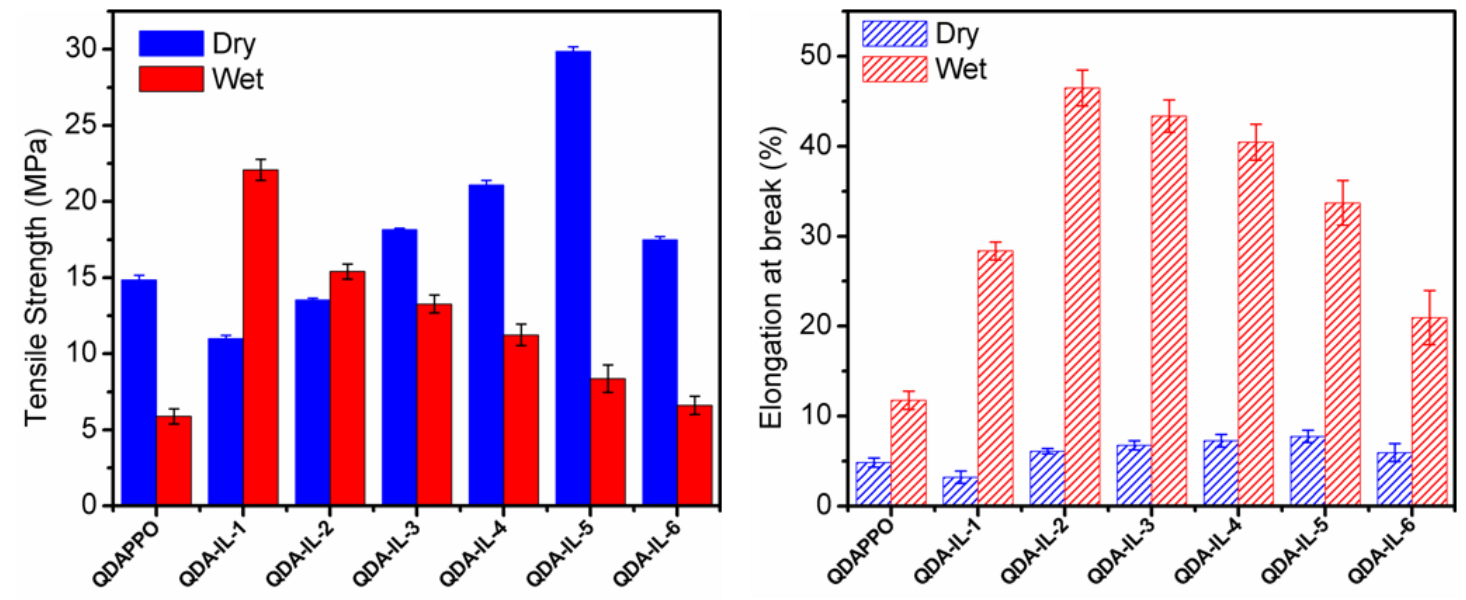

Figure 8: Mechanical properties of QDAPPO and QDA-IL membranes in both dry and wet states; a) Tensile strength (TS), b) Elongation at break (EB)

\section{Conclusion}

Highly charge hierarchical porous anion exchange membranes were successful prepared by crosslinking quaternized poly (2-dimethylaminoethanol-N-2,3dimethylphenyl oxide) and 3-methyl-1-(3-(triethoxysilyl)propyl)-1H-imidazolium chloride (Ionic liquid, IL). The obtained membranes are thin, mechanically stable and possess high ion exchange capacity for versatile anion exchange membranes based applications. In diffusion dialysis for acid recovery, the hierarchical porous membranes exhibited high performance, excellent membrane selectivity and superior acid recovery than the commercial DF-120 membranes and many others reported anion exchange membranes. We found that the proton diffusion coefficient increases with temperature up to $0.045 \mathrm{~m} \mathrm{~h}^{-1}$ at $65^{\circ} \mathrm{C}$. These membranes are mechanically and thermally stable. This work presents a feasible strategy to construct gradient porous system, highly stable and high performance anion exchange membranes with controllable morphology. Therefore, by improving membrane composition and adjust pores size, ongoing study aim to expand application in salinity gradient energy harvesting techniques, desalination and fuel cells.

\section{Acknowledgements}

This research is supported in part by the National Science Foundation of China (Nos. 91534203, 21490581) and One Hundred Person Project of the Chinese Academy of Sciences. The first author thanks the CAS-TWAS President's fellowship for PhD programs. 


\section{References}

[1] Bramer HC. Pollution control in the steel industry. Environmental Science \& Technology. 1971;5:1004-8.

[2] Dean JG, Bosqui FL, Lanouette KH. Removing heavy metals from waste water.

Environmental Science \& Technology. 1972;6:518-22.

[3] Wisniewski J, Suder S. Water recovery from etching effluents for the purpose of rinsing stainless steel. Desalination. 1995;101:245-53.

[4] Boucher M, Turcotte N, Guillemette V, Lantagne G, Chapotot A, Pourcelly G, et al.

Recovery of spent acid by electrodialysis in the zinc hydrometallurgy industry: Performance study of different cation-exchange membranes. Hydrometallurgy. 1997;45:137-60.

[5] Wiśniewski J, Wiśniewska G. Water and acid recovery from the rinse after metal etching operations. Hydrometallurgy. 1999;53:105-19.

[6] Xue M, Kendall A, Xu Z, Schoenung JM. Waste Management of Printed Wiring Boards: A Life Cycle Assessment of the Metals Recycling Chain from Liberation through Refining. Environmental Science \& Technology. 2015;49:940-7.

[7] Gottliebsen K, Grinbaum B, Chen D, Stevens GW. Recovery of sulfuric acid from copper tank house electrolyte bleeds. Hydrometallurgy. 2000;56:293-307.

[8] Agrawal A, Kumari S, Ray BC, Sahu KK. Extraction of acid and iron values from sulphate waste pickle liquor of a steel industry by solvent extraction route. Hydrometallurgy. 2007;88:58-66.

[9] Kesieme UK, Aral H, Duke M, Milne N, Cheng CY. Recovery of sulphuric acid from waste and process solutions using solvent extraction. Hydrometallurgy. 2013;138:14-20.

[10] Petruzzelli D, Petrella M, Boghetich G, Calabrese P, Petruzzelli V, Petrella A. Neutralization of Acidic Wastewater by the Use of Waste Limestone from the Marble Industry. Mechanistic Aspects and Mass Transfer Phenomena of the Acid-Base Reaction at the Liquid-Solid Interface. Industrial \& Engineering Chemistry Research. 2009;48:399-405.

[11] Kobuchi Y, Motomura H, Noma Y, Hanada F. Application of ion exchange membranes to the recovery of acids by diffusion dialysis. Journal of Membrane Science. 1986;27:173-9.

[12] Tomaszewska M, Gryta M, Morawski AW. Recovery of hydrochloric acid from metal pickling solutions by membrane distillation. Separation and Purification Technology. 2001;22-23:591-600.

[13] Gryta M, Tomaszewska M, Karakulski K. Wastewater treatment by membrane distillation. Desalination. 2006;198:67-73.

[14] Urano K, Ase T, Naito Y. Recovery of acid from wastewater by electrodialysis. Desalination. 1984;51:213-26.

[15] Liu H, Ramnarayanan R, Logan BE. Production of Electricity during Wastewater Treatment Using a Single Chamber Microbial Fuel Cell. Environmental Science \& Technology. 2004;38:2281-5.

[16] Xu T. Ion exchange membranes: State of their development and perspective. Journal of Membrane Science. 2005;263:1-29.

[17] Logan BE, Elimelech M. Membrane-based processes for sustainable power generation using water. Nature. 2012;488:313-9.

[18] German M, SenGupta AK, Greenleaf J. Hydrogen Ion $(\mathrm{H}+)$ in Waste Acid as a Driver for Environmentally Sustainable Processes: Opportunities and Challenges. Environmental Science \& Technology. 2013;47:2145-50.

[19] Stachera DM, F. Childs R, Mika AM, Dickson JM. Acid recovery using diffusion dialysis with poly(4-vinylpyridine)-filled microporous membranes. Journal of Membrane Science. 1998;148:119-27.

[20] Sridhar P, Subramaniam G. Recovery of acid from cation exchange resin regeneration waste by diffusion dialysis. Journal of Membrane Science. 1989;45:273-80. 
[21] Jung Oh S, Moon S-H, Davis T. Effects of metal ions on diffusion dialysis of inorganic acids. Journal of Membrane Science. 2000;169:95-105.

[22] Varcoe JR, Atanassov P, Dekel DR, Herring AM, Hickner MA, Kohl PA, et al. Anionexchange membranes in electrochemical energy systems. Energy \& Environmental Science. 2014;7:3135-91.

[23] Ran J, Wu L, Ru Y, Hu M, Din L, Xu T. Anion exchange membranes (AEMs) based on poly(2,6-dimethyl-1,4-phenylene oxide) (PPO) and its derivatives. Polymer Chemistry. 2015;6:5809-26.

[24] Couture G, Alaaeddine A, Boschet F, Ameduri B. Polymeric materials as anion-exchange membranes for alkaline fuel cells. Progress in Polymer Science. 2011;36:1521-57.

[25] Guler E, Zhang Y, Saakes M, Nijmeijer K. Tailor-Made Anion-Exchange Membranes for Salinity Gradient Power Generation Using Reverse Electrodialysis. ChemSusChem. 2012;5:2262-70.

[26] Yan J, Hickner MA. Anion Exchange Membranes by Bromination of BenzylmethylContaining Poly(sulfone)s. Macromolecules. 2010;43:2349-56.

[27] Li X, Yu Y, Liu Q, Meng Y. Synthesis and Properties of Anion Conductive lonomers Containing Tetraphenyl Methane Moieties. ACS Applied Materials \& Interfaces.

2012;4:3627-35.

[28] He SS, Strickler AL, Frank CW. A Semi-Interpenetrating Network Approach for Dimensionally Stabilizing Highly-Charged Anion Exchange Membranes for Alkaline Fuel Cells. ChemSusChem. 2015;8:1472-83.

[29] Gu S, Skovgard J, Yan YS. Engineering the Van der Waals Interaction in Cross-LinkingFree Hydroxide Exchange Membranes for Low Swelling and High Conductivity.

ChemSusChem. 2012;5:843-8.

[30] Lin B, Qiu L, Lu J, Yan F. Cross-Linked Alkaline lonic Liquid-Based Polymer Electrolytes for Alkaline Fuel Cell Applications. Chemistry of Materials. 2010;22:6718-25.

[31] Ran J, Wu L, Wei B, Chen Y, Xu T. Simultaneous Enhancements of Conductivity and Stability for Anion Exchange Membranes (AEMs) through Precise Structure Design. Scientific Reports. 2014;4:6486.

[32] Li N, Yan T, Li Z, Thurn-Albrecht T, Binder WH. Comb-shaped polymers to enhance hydroxide transport in anion exchange membranes. Energy \& Environmental Science. 2012;5:7888-92.

[33] Tongwen $X$, Weihua Y. Fundamental studies of a new series of anion exchange membranes: membrane preparation and characterization. Journal of Membrane Science. 2001;190:159-66.

[34] Tongwen $X$, Weihua $Y$. Tuning the diffusion dialysis performance by surface cross-linking of PPO anion exchange membranes - simultaneous recovery of sulfuric acid and nickel from electrolysis spent liquor of relatively low acid concentration. Journal of Hazardous Materials. 2004;109:157-64.

[35] Wu Y, Luo J, Zhao L, Zhang G, Wu C, Xu T. QPPO/PVA anion exchange hybrid membranes from double crosslinking agents for acid recovery. Journal of Membrane Science.

2013;428:95-103.

[36] Maes AM, Pandey TP, Vandiver MA, Lundquist LK, Yang Y, Horan JL, et al. Preparation and characterization of an alkaline anion exchange membrane from chlorinated poly(propylene) aminated with branched poly(ethyleneimine). Electrochimica Acta. 2013;110:260-6.

[37] Wang L, Hickner MA. Low-temperature crosslinking of anion exchange membranes. Polymer Chemistry. 2014;5:2928-35.

[38] Pandey AK, Childs RF, West M, Lott JNA, McCarry BE, Dickson JM. Formation of porefilled ion-exchange membranes with in situ crosslinking: Poly(vinylbenzyl ammonium salt)filled membranes. Journal of Polymer Science Part A: Polymer Chemistry. 2001;39:807-20. 
[39] Xu T, Liu Z, Huang C, Wu Y, Wu L, Yang W. Preparation of a Novel Hollow-Fiber AnionExchange Membrane and Its Preliminary Performance in Diffusion Dialysis. Industrial \& Engineering Chemistry Research. 2008;47:6204-10.

[40] Sun F, Wu C, Wu Y, Xu T. Porous BPPO-based membranes modified by multisilicon copolymer for application in diffusion dialysis. Journal of Membrane Science. 2014;450:10310.

[41] Lin X, Shamsaei E, Kong B, Liu JZ, Xu T, Wang H. Fabrication of asymmetrical diffusion dialysis membranes for rapid acid recovery with high purity. Journal of Materials Chemistry A. 2015;3:24000-7.

[42] Lin X, Shamsaei E, Kong B, Liu JZ, Hu Y, Xu T, et al. Porous diffusion dialysis membranes for rapid acid recovery. Journal of Membrane Science. 2016;502:76-83.

[43] Zhao Q, Yin M, Zhang AP, Prescher S, Antonietti M, Yuan J. Hierarchically Structured Nanoporous Poly(Ionic Liquid) Membranes: Facile Preparation and Application in Fiber-Optic pH Sensing. Journal of the American Chemical Society. 2013;135:5549-52.

[44] Zhang K, Feng X, Sui X, Hempenius MA, Vancso GJ. Breathing Pores on Command: Redox-Responsive Spongy Membranes from Poly(ferrocenylsilane)s. Angewandte Chemie International Edition. 2014;53:13789-93.

[45] Täuber K, Zhao Q, Antonietti M, Yuan J. Tuning the Pore Size in Gradient Poly(ionic liquid) Membranes by Small Organic Acids. ACS Macro Letters. 2015;4:39-42.

[46] Zhang Z, Zhao Q, Yuan J, Antonietti M, Huang F. A hybrid porous material from a pillar[5]arene and a poly(ionic liquid): selective adsorption of $n$-alkylene diols. Chemical Communications. 2014;50:2595-7.

[47] Brenna S, Posset T, Furrer J, Blümel J. 14N NMR and Two-Dimensional Suspension $1 \mathrm{H}$ and 13C HRMAS NMR Spectroscopy of Ionic Liquids Immobilized on Silica. Chemistry - A European Journal. 2006;12:2880-8.

[48] Wu C, Wu Y, Luo J, Xu T, Fu Y. Anion exchange hybrid membranes from PVA and multialkoxy silicon copolymer tailored for diffusion dialysis process. Journal of Membrane Science. 2010;356:96-104.

[49] Innocenzi P. Infrared spectroscopy of sol-gel derived silica-based films: a spectramicrostructure overview. Journal of Non-Crystalline Solids. 2003;316:309-19.

[50] Fidalgo A, Ilharco LM. The defect structure of sol-gel-derived silica/polytetrahydrofuran hybrid films by FTIR. Journal of Non-Crystalline Solids. 2001;283:144-54.

[51] Wu Y, Wu C, Xu T, Fu Y. Novel anion-exchange organic-inorganic hybrid membranes prepared through sol-gel reaction of multi-alkoxy precursors. Journal of Membrane Science. 2009;329:236-45.

[52] Luo J, Wu C, Wu Y, Xu T. Diffusion dialysis of hydrochloride acid at different temperatures using PPO-SiO 2 hybrid anion exchange membranes. Journal of Membrane Science. 2010;347:240-9.

[53] Wu Y, Wu C, Li Y, Xu T, Fu Y. PVA-silica anion-exchange hybrid membranes prepared through a copolymer crosslinking agent. Journal of Membrane Science. 2010;350:322-32. [54] Wang C, Wu C, Wu Y, Gu J, Xu T. Polyelectrolyte complex/PVA membranes for diffusion dialysis. Journal of Hazardous Materials. 2013;261:114-22.

[55] Tugas I, Pourcelly G, Gavach C. Electrotransport of protons and chloride ions in anion exchange membranes for the recovery of acids. Part I. Equilibrium properties. Journal of Membrane Science. 1993;85:183-94.

[56] Daiko Y, Kasuga T, Nogami M. Pore size effect on proton transfer in sol-gel porous silica glasses. Microporous and Mesoporous Materials. 2004;69:149-55.

[57] Ran J, Wu L, Varcoe JR, Ong AL, Poynton SD, Xu T. Development of imidazolium-type alkaline anion exchange membranes for fuel cell application. Journal of Membrane Science. 2012;415-416:242-9. 
